\title{
La responsabilidad como valor esencial durante la formación escolar ${ }^{1}$
}

\section{Responsibility as an essential value during school training}

DOI: http://dx.doi.org/10.17981/cultedusoc.9.3.2018.58

\author{
Artículo de investigación. Fecha de recepción: 15/06/2018. Fecha de aceptación: 27/11/2018 \\ Liliana Escudero'; \\ Edith Velasco y Juan Palmera ${ }^{3}$ \\ IED San José de Pueblo Viejo, Sede Principal (Colombia) \\ liaescudero24@gmail.com
}

Para citar este artículo:

Escudero, L., Velasco, E. y Palmera, J. (2018). La responsabilidad como valor esencial durante la formación escolar. Cultura. Educación y Sociedad 9(3), 493-498. DOI: http://dx.doi.org/10.17981/cultedusoc.9.3.2018.58

\section{Resumen}

La responsabilidad es considerado un valor del ser humano, que se caracteriza por la capacidad del individuo de actuar de la manera correcta (o de acuerdo a lo esperado) y comprometerse con propósitos conjuntos. Este estudio tuvo como objetivo fomentar la responsabilidad como valor esencial durante la formación escolar. Metodológicamente se orientó bajo el enfoque cualitativo, utilizando el tipo de investigación acción-participación. Para la recogida de datos se utilizó un diario de campo, formatos de entrevistas y encuestas. La unidad de análisis estuvo conformada por ciento treinta (130) estudiantes con edades que oscilaron entre los cuatro (12) y doce (16) años, de grados comprendidos sexto y decimo de la IED San José de Pueblo Viejo, Magdalena. Los resultados arrojaron que los estudiantes que participaron en el proyecto mostraron mejoría en la responsabilidad, aspecto importante para lograr un buen rendimiento académico.

Palabras clave: responsabilidad, valores, formación escolar.

\section{Abstract}

The responsibility to consider a quality and a value of the human being, is a positive characteristic of the people who can be forced and act correctly. The objective of this study was to promote responsibility as a fundamental value during school education. Methodologically, it was guided by the qualitative approach, using the type of action-participation research. A field diary, interview formats and surveys are used to collect data. The unit of analysis was formed with one hundred and thirty (130) students aged between four (12) and twelve (16) years, of grades comprised sixth and tenth of the IED San José de Pueblo Viejo, Magdalena. The results showed that the students who participated in the project were better at the responsibility, an important aspect to achieve a good academic performance.

Keywords: responsibility, values, school education.

1 Este artículo ha sido derivado del Programa de Fortalecimiento de la Cultura Ciudadana y Democrática CT+I a través de la IEP apoyada en TIC en el Departamento de Magdalena: CICLON

2 Líder del Grupo de investigación "Los Súper Responsables".

3 Docentes del Grupo de investigación "Los Súper Responsables" de la IED San José de Pueblo Viejo, Sede Principal

- The author; licensee Universidad de la Costa - CUC.

Cultura, Educación y Sociedad vol. 9 no. 3, pp. 493-498. Diciembre, 2018

Barranquilla. ISSN 2389-7724 Online 


\section{Introducción}

La necesidad educativa de fomentar la cooperación entre las familias y las escuelas, es importante a partir de los efectos positivos que conlleva tanto para estudiantes, padres de familia, docentes, como para la escuela y la comunidad en la que tiene lugar. Fortalecer o desarrollar el valor de la responsabilidad en el ámbito educativo, va de la mano con la acción de cumplir con las obligaciones personales, familiares, laborales y ciudadanas; con rendir cuentas; con obedecer incluso a nuestra propia conciencia y a las autoridades (Febres, 2007).

Es importante mencionar que la falta de responsabilidad es un problema que se encuentra presente en muchos estudiantes de la IED San José, y se evidencia en acciones como el incumplimiento de los compromisos establecidos por las autoridades respectivas (directivos o docentes), mantener al margen las actividades impuestas y en vez de ello atenerse a compañeros cercanos. Es por ello, que surge la necesidad de preparar a los estudiantes, para que aprendan que esos quehaceres son de obligatorio cumplimiento para que obtenga resultados óptimos en su rol de estudiante y la vida diaria.

De acuerdo a Sánchez (2018) la responsabilidad significa hacerse cargo de lo que se ofrece hacer y de las obligaciones que son asignadas por el rol que se tiene en la sociedad, y en este sentido una persona responsable es capaz de tomar decisiones conscientemente, aceptando las consecuencias de sus actos y de sus propias decisiones.

En este entendido, la responsabilidad social, "no reclama sólo la ética individual de las personas que componen las organizaciones, sino que ven un ente con responsabilidad propia económica, social y medioambiental a la que debe exigírsele comportamientos acordes a la misma" (Hernández y Jiménez, 2015, p. 11).

Desde la ética, la responsabilidad ha sido asumida como un concepto clave, bien sea por cuestiones psicológicas, jurídicas, semánticas o filosóficas; cobrando cierto protagonismo en las reflexiones e investigaciones actuales, sobre todo en lo que respecta al impacto hacia el ámbito profesional (Sánchez, 2008).

Lo anterior en el entendido de que la humanidad debe ser formada como sociedad de líderes éticos e integrales que puedan emplear habilidades para consensuar entre miembros de grupos y dirigir acciones por y para un bien común de países, organismos, instituciones entre otros (Kadi y Acevedo, 2014)

Dentro de la diversidad de las relaciones humanas, se puede pensar en la responsabilidad desplegada en al menos tres aspectos, diferenciados, pero también entrecruzados (Friedman, 1970). La moralidad es construida teniendo en cuenta un entramado de interrelaciones que se van construyendo en el trascurso de la vida, las cuales permiten al individuo adoptar posturas críticas y reflexivas sobre diversas cuestiones (Vallaeys, 2008).

Para desarrollar el sentido de la responsabilidad y que este valor crezca dentro de cada individuo, es necesario revisar tareas y deberes, despertar el sentimiento de superación, y ayudar para que lo demás también logre ser más responsables cada día. (Sánchez, 1995). En ese sentido, es posible argumentar que tener responsabilidad implica asumir una postura mental en la que se integren el conocimiento, dominio interior y seguridad y confianza de dar lo mejor de cada uno en cada circunstancia, sin importar la situación (Febres, 2007). La responsabilidad y la libertad deben ir de la mano, pues si no se goza de libertad absoluta y completa no se logrará desarrollar el sentido de la responsabilidad y no se podrá responder a los actos sean buenos o malos (Fraile, 2002).

La libertad es simplemente el espacio en el que un hombre puede actuar sin ser obstaculizado por otros, un espacio libre de imposiciones (Berlín, 2001). Ahora bien, es posible argumentar que toda escuela tiene la necesidad de ser un centro de libertad de 
aprendizaje, en donde sea posible valorar los aspectos positivos de estudiantes y profesores (Foucault, 1984).

Es posible asumir además, que los individuos que comparten diversidad de niveles de enseñanza, cualidades, intereses intelectuales y comportamientos, pueden generar espacios de libertad en entornos de aprendizaje. Resultados de investigaciones indican que los estudiantes responden con una avidez por aprender, con una creciente confianza en sí mismos, con independencia y con energía creadora (Rogers, 1996). Donde ser totalmente humano, confiar en las personas que nos rodean y conceder libertad con responsabilidad, no son cosas fáciles de lograr (Sen, 2002).

Quienes investigan, piensan que es importante entender que la capacidad de responsabilidad, en estudiantes en formación va de la mano con la enseñanza y costumbres aprendidas tanto en la escuela como en el hogar, el que un estudiante presente cualidades de irresponsabilidad es un hecho corregible, sería suficiente con un buen seguimiento de sus actitudes y comportamientos para un posterior cambio.

Formar en valores implica, educar al estudiante para que encuentre sentido en sus acciones, responsabilizándose por las mismas, lo cual le permitirá tomar decisiones asertivas, desarrollar capacidades como solución de conflictos (Arrieta, 2016), pensamiento crítico, que a su vez le posibilite plantear objetivos, para buscar la manera más idónea de abordar la realidad, presentándole al educando una formación con una dimensión humana e integral, que permita el desarrollo libre de su personalidad, partiendo de la comprensión de los derechos del otro (Conejo, 2012).

Actualmente la formación en valores es una de las temáticas más estudiadas en el debate educativo, debido al deterioro en materia de conciencia ciudadana observado en todos los niveles sociales, evidenciando conductas como discriminación, inconformidad social, violencia en sus distintas manifes- taciones y con múliples actores (familiar, personal, escolar). Por lo anterior, la escuela mediante proyectos curriculares ha intentado impulsar la formación en valores, no obstante, se convierte en un reto para la educación básica, donde se entiende que este tipo de enseñanza debe estructurarse más allá de planteamientos teóricos (Díaz, 2006).

Por consiguiente, contener dentro de la malla curricular la asignatura de ética y valores, no es garantía de realizar un proceso de formación en valores adecuado que presente un impacto positivo en la comunidad educativa, puesto que se hace necesario transversalizar dicha área a las diferentes asignaturas integrándola al proceso de enseñanza - aprendizaje, esto se logra cuando los estudiantes se conviertan en individuos capaces de entender y transformar la realidad, adquiriendo conocimientos, pero a la vez, analizando a fondo los fenómenos que suceden en la vida cotidiana (Botero, 2006).

Para formar ciudadanos integrales, con competencias del ser es necesario diseñar estrategias pedagógicas basadas en valores, que permitan a los estudiantes relacionarse con los demás y adquirir progresivamente pautas básicas de convivencia, necesarias para relacionarse en sociedad, así como prepararse para la solución pacífica de conflictos y para el ejercicio activo de la ciudadanía, siendo tolerante frente las diferencias, respetando los derechos humanos y el pluralismo propio de una sociedad democrática (Conejo, 2012).

Sarre (2014), define el valor escolar como un proceso mediante el que es posible incorporar normas éticas en procesos habituales de aprendizaje. Una actividad que se origina en niveles de enseñanza tanto formal como informal. Para quienes investigan los valores escolares son aquellas normas y conductas que orientan a los niños en etapa escolar. Considerándose como creencias que le permiten al estudiante tener un comportamiento adecuado en su entorno social, llevándolo hacia el crecimiento y realización como persona. 
En resumen, se debe concebir la formación desde una visión integral que atienda la esencia del ser en su multidimensionalidad, observando la educación como proceso formativo, que requiere un análisis amplio, en cuyo plano normativo-operativo se conceptualice una visión de integralidad, reflejo del perfil de competencias que se persigue consolidar (Marín, Cabas, Cabas y Paredes, 2018), y poder avanzar en el conocimiento (Maculan, Jiménez y Castellanos, 2015)

\section{Metodología}

\section{Diseño}

Se realizó un estudio de tipo cualitativo, utilizando el modelo de investigación acciónparticipación. La investigación cualitativa busca conocer e interpretar la realidad de los participantes a través de sus propias experiencias, entregando una información subjetiva del fenómeno de estudio (Hernández, Fernández y Baptista, 2010).

Por su parte el modelo de investigación acción-participación, plantea una producción de conocimiento basado en la reflexión de los participantes, teniendo en cuenta la participación activa de maestros en el proceso de enseñanza y acompañamiento tecnológico, guiando al estudiante en su proceso de investigación. Generando nuevo saber y conocimiento sobre una realidad determinada en un proceso de aprendizaje colaborativo. Colmenares (2012) manifiesta que este modelo constituye una opción metodológica de mucha riqueza ya que permite por una parte la expansión del conocimiento, temática de interés o situación problemática, brindando a la vez una alternativa de cambio hacia la transformación, implicando incluso una nueva visión del hombre y de la comunidad inmersa.

\section{Participantes}

La unidad de análisis estuvo conformada por ciento treinta (130) estudiantes de la IED "San José" de Pueblo Viejo, distribuidos en los cursos desde preescolar hasta quinto de primaria, con edades que oscilaron entre los doce (12) y dieciséis (16) años. Los estudiantes participantes, fueron aquellos quienes sus acudientes devolvieron debidamente firmado el consentimiento informado.

\section{Técnicas e instrumentos}

Se seleccionaron técnicas e instrumentos de recolección de información propios de la investigación cualitativa, tales como, observación participante y diario de campo, entrevistas y encuestas, donde se consignaron todas las experiencias pedagógicas, observadas en el proceso de fortalecimiento de la responsabilidad como valor esencial durante la formación escolar.

\section{Procedimiento}

La metodología del proyecto de investigación se llevó a cabo a partir unas fases, inicialmente se realizó un diseño de estrategias pedagógicas, basadas en la investigación y teniendo en cuenta las TIC; posteriormente se realizó una implementación y transversalización de las estrategias diseñadas con el objeto de fortalecer la responsabilidad, en el aula de clase por parte del cuerpo docente. En un tercer momento se realizó un análisis e interpretación de resultados, culminando con la reflexión y apropiación social sobre los conocimientos generados.

\section{Resultados}

Una vez realizada la aplicación de instrumentos, se procesa todo el conjunto de información recogida, en dos fases: en un primer momento se preparan los datos transcribiéndolos y organizándolos y en un segundo momento se extraen elementos analíticos e interpretativos, con la participación de la comunidad en este proceso (Martí, 2017).

Los sujetos de análisis fueron los estudiantes con ayuda de las observaciones y registros que iba realizando adicionalmente el cuerpo docente. Para la comunidad 
educativa fue importante esta investigación pues la responsabilidad como valor escolar de acuerdo a Sarre (2014) hace parte de las pautas morales para una convivencia orientada en principios y valores humanos. Durante el proceso, se buscó describir y comprender lo sucedido durante las experiencias pedagógicas, explicar los resultados de tales experiencias y razones por las que se obtuvieron, hacer reflexiones a los procesos a partir de los roles que cada uno cumple e identificar estrategias desde la comunidad educativa para mejorar las experiencias futuras.

Los estudiantes que participaron en el proyecto mejoraron su capacidad de ser responsables y por consiguiente evidenciaron mejoría académicamente, convirtiéndose así en ejemplo del resto de sus compañeros; por lo tanto, se afirma que arrojó buenos resultados. A través de métodos de observación, realización de entrevistas y encuestas se pudo lograr crear una concientización en los alumnos, incluso existiendo de cierta manera de acuerdo a lo dicho por los estudiantes, repercusiones entre padres de familias y acudientes. Puede manifestarse que el grupo de estudiantes participantes son más responsables, más consientes, dicho valor en los estudiantes, era cuestión de intervención y preocupación por parte de los superiores. A continuación, se presenta la sistematización teórica auto observable de los valores escolares mediante la IEP.

\section{Discusión}

La responsabilidad es un valor que se adopta a medida que vamos creciendo, la educación y las costumbres que nos enseñen las personas que nos rodean nos ayudara a direccionar este valor, más que a un simple conocimiento, a la práctica. Lo anterior se puede sustentar en los planteamientos de Conejo, (2012) quien manifiesta que formar en valores implica, educar al estudiante para que encuentre sentido en sus acciones, responsabilizándose por las mismas, lo cual le permitirá tomar decisiones asertivas, desarrollar capacidades como solución de conflictos, pensamiento crítico, que a su vez le posibilite plantear objetivos, para buscar la manera más idónea de abordar la realidad, presentándole al educando una formación con una dimensión humana e integral, que permita el desarrollo libre de su personalidad, partiendo de la comprensión de los derechos del otro.

El problema por falta de responsabilidad, es un problema tanto en la vida cotidiana como en las escuelas, problema que, si se interviene con tiempo y de forma adecuada, podrá dejar de ser un obstáculo en el desarrollo de los seres humanos, y en caso de los estudiantes, permitiría desarrollar habilidades que los direccionarían a ser personas de mente abierta y con un gran potencial intelectual. Estos resultados confluyen con los planteamientos de Díaz, (2006) y Herrera-Mendoza y Rico, (2014). quienes expresan que actualmente la formación en valores es una de las temáticas más estudiadas en el debate educativo, debido al deterioro en materia de conciencia ciudadana observado en todos los niveles sociales, evidenciando conductas negativas en los diversos grupos sociales. Por lo anterior, la escuela mediante proyectos curriculares ha intentado impulsar la formación en valores, no obstante, se convierte en un reto para la educación básica, donde se entiende que este tipo de enseñanza debe estructurarse más allá de planteamientos teóricos.

\section{Referencias}

Arrieta, M. (2016). Los masones en el mundo: Geopolítica masónica. La historia de los hijos de la luz. Córdoba: Almuzara.

Berlin, I. (2001). Dos conceptos de libertad y otros escritos. Madrid: Alianza.

Botero, C. (2006). Los ejes transversales como instrumento pedagógico para la formación en valores. Revista Politécnica. 2(3). 
Colmenares, A. (2012). Investigación-acción participativa: una metodología integradora del conocimiento y la acción. Voces y Silencios, 3(1). 102-115.

Conejo, P. (2012). El valor formativo de la música para la educación en valores. $R e$ vista de Educação e Humanidades, 2, 263-278.

Corral, H. (2010). Concurrencia de acciones de responsabilidad civil contractual y extracontractual en los daños causados por accidentes del trabajo. Revista chilena de derecho privado, (14), 69-107.

Chomsky, N. (1969). La responsabilidad de los intelectuales. Buenos Aires: Galerna.

Díaz, A. (2006). La educación en valores: Avatares del currículum formal, oculto y los temas transversales. Revista electrónica de investigación educativa. 8(1). 1-15. Recuperado de https://redie.uabc.mx/redie/article/view/117/201

Foucault, M. (1984). La ética del cuidado de uno mismo como práctica de la libertad. Hermenéutica del sujeto, 107.

Friedman, M. (1970). La responsabilidad social de las empresas es incrementar sus beneficios. The New York Times Magazine, 13.

Hernández, H. y Jiménez, A. (2015). Responsabilidad social empresarial, zona urbana del distrito de Barranquilla: estudio etnográfico. Económicas CUC, 36(2), 9-18.

Hernández R, Fernández C. y Baptista M. (2014). Metodología de la investigación. México, D.F.: McGraw - Hill.

Herrera-Mendoza, K. y Rico, R. (2014). El Clima Escolar Como Elemento Fundamental de la Convivencia en la Escuela. Revista escenarios, 12(2). 7-18.

Kadi, O. y Acevedo, Á. (2014). Liderazgo ético frente a la diversidad cultural dentro de las organizaciones con régimen disciplinario. Económicas CUC, 35(2), 75-88.
Maculan, A, Jiménez, C. y Castellanos, O. (2015). Aprendizaje en el proceso de incubación de empresas de base tecnológica. Económicas CUC, 36(1), 15-48.

Marín, F., Cabas, L., Cabas, L. y Paredes, A. (2018). Formación Integral en Profesionales de la Ingeniería. Análisis en el Plano de la Calidad Educativa. Formación universitaria, 11(1), 13-24.

Martí, J. (2017). La investigación-acción participativa: estructura y fases. Recuperado de http://www.redcimas.org/wordpress/ wp-content/uploads/2012/08/m_JMarti_ IAPFASES.pdf

Popper, K. (1988). Tolerancia y responsabilidad intelectual. Sociedad abierta, universo abierto. Madrid: Tecnos.

Rogers, C., Freiberg, H. y Soler, S. (1996). Libertad y creatividad en la educación. Buenos Aires: Paidós.

Sánchez, P. (2018) Responsabilidad: ¿Qué es? Concepto y claves para ser más responsable. Cognifit: Salud, cerebro y neurociencia.

Sánchez, M. (2008). Ética y profesión: la responsabilidad en términos de prudencia responsable. El caso de la Psicología. Fundamentos en Humanidades, 9(17). 145161.

Sarre, P. (2014). El debate sobre los valores en la escuela. México, D.F.: Fondo de Cultura Económica.

Sen, A. (2000). El desarrollo como libertad. Gaceta Ecológica, (55). 14-20.

Vallaeys, F. (2008). "Responsabilidad Social Universitaria": una nueva filosofía de gestión ética e inteligentes para las universidades. Revista Educación Superior y Sociedad 13(2), 191-220.

Wester, J. (2008). Dimensiones y retos de una educación para la responsabilidad ciudadana. Utopía y praxis latinoamericana, 13(42), 55-69. 